S. Ewig ${ }^{1}$ T. Schaberg ${ }^{2}$

\section{Ein neues Konzept der antimikrobiellen Therapie von Patienten mit akuter Exazerbation der chronisch- obstruktiven Lungenerkrankung (COPD)}

A New Concept for the Treatment of Patients with Acute Exacerbations of Chronic Obstructive Pulmonary Disease (COPD)

\section{Zusammenfassung}

In der Praxis stellt die antimikrobielle Therapie einen festen Bestandteil in der Behandlung von Patienten mit akuter Exazerbation der COPD dar. Demgegenüber ist die Datenlage für eine solche Therapie sehr begrenzt. Die meisten Plazebo-kontrollierten Studien entsprechen nicht mehr den methodischen Standards, die aktuell gefordert werden müssen. Aus den verfügbaren Daten wird ein Konzept der antimikrobiellen Therapie entwickelt, das zu einer restriktiven Indikationsstellung für eine solche Therapie anleitet. Die wichtigsten Punkte dieses Konzepts umfassen dabei die Orientierung des Behandlungsortes sowie der Indikation zur antimikrobiellen Therapie am Schweregrad der akuten Exazerbation (nicht primär der COPD) sowie am Typ der Exazerbation (Typ I nach Anthonisen). Die Differenzialindikation erfolgt entsprechend einer intraindividuellen Rotation zur Begrenzung des antimikrobiellen Selektionsdrucks. Das vorgestellte Konzept kann gleichzeitig heute schon klinische Anwendung finden und einen Rahmen für die Bearbeitung offener Fragen in künftigen Studien darstellen.

\section{Abstract}

In practice, antimicrobial treatment is usually thought to be regularly indicated in the treatment of patients with acute exacerbations of COPD. However, data supporting this practice are very limited. Most placebo-controlled studies do not satisfy currently required methodological quality standards. We develop a concept of restrictive indications for antimicrobial treatment from the available data. Cornerstones of this concept include the selection of treatment setting as well as the indication for antimicrobial treatment according to the severity of the acute exacerbation (not primarily of COPD) and to the type of exacerbation (Anthonisen type I). The selection of antimicrobial agents is based on an intraindividual rotation concept in order to limit antimicrobial selection pressure. The concept presented here can at the same time be used as a clinical guide as well as a framework for future studies dealing with currently unresolved issues.

\section{Einleitung}

In allen aktuellen Leitlinien von Fachgesellschaften gehört neben der bronchodilatatorischen Inhalationstherapie sowie der antiinflammatorischen Steroidtherapie die antimikrobielle Therapie zum festen Bestandteil des Behandlungskonzepts der akuten Exazerbation einer chronisch-obstruktiven Lungenerkrankung
(COPD) [1 - 4]. Die sehr begrenzte Evidenz für die Notwendigkeit bzw. Wirksamkeit der antimikrobiellen Therapie wird hingegen nicht immer ausreichend herausgestellt. Die Leitlinie der ERS (3) sowie die deutschsprachige S3-Leitlinie der PEG, DGP und DGAI (4), an denen die Autoren mitgewirkt haben, sprechen bereits sehr differenzierte Empfehlungen aus. Aus klinischer Sicht bleiben jedoch vor allem konzeptuell eine Reihe von Fragen of- 
fen. Diese betreffen vor allem die Indikation zur antimikrobiellen Therapie, den Zusammenhang der Schweregrade der COPD mit den Schweregraden der akuten Exazerbation als Basis für differenzialtherapeutische Entscheidungen sowie Strategien zur Limitation des Selektionsdrucks.

Tatsächlich ist der Mangel aussagekräftiger Studien über eine der häufigsten Atemwegserkrankungen mit beträchtlichen Folgekosten ein fortgesetztes Ärgernis. Im Ergebnis begründet dies einen häufig unkritischen Einsatz antimikrobieller Substanzen, der als eine wichtige Ursache für weltweit steigende Resistenzraten bakterieller Erreger angesehen werden muss.

Im Folgenden soll daher ein Konzept der antimikrobiellen Therapie der akuten Exazerbation der COPD dargelegt werden, das in einer kritischen Sicht der verfügbaren Daten gründet und zu einer restriktiven Indikationsstellung zur antimikrobiellen Therapie anleitet. Dieses bewusst pragmatisch formulierte Konzept kann dabei gleichzeitig heute schon klinische Anwendung finden und einen Rahmen für die Bearbeitung offener Fragen in künftigen Studien darstellen.

\section{Rolle der Infektion in der akuten Exazerbation}

In einer Reihe von Studien konnte nachgewiesen werden, dass Patienten mit COPD häufig eine tracheobronchiale Kolonisation aufweisen. In Studien mit bronchoskopischer Untersuchungstechnik wurde dabei eine Kolonisation in ca. 25\% der Fälle nachgewiesen [5 - 9]. Häufigste Erreger waren dabei Haemophilus influenzae und Streptococcus pneumoniae. Patienten mit einer tracheobronchialen Kolonisation weisen eine chronische inflammatorische Reaktion im bronchopulmonalen Kompartiment auf, die sich in erhöhten Spiegeln einer Reihe von inflammatorischen Mediatoren (z. B. Interleukin-8, TNF- $\alpha$, MPO) im Sputum und/ oder der BALF reflektiert [5]. Patienten mit einer tracheobronchialen Kolonisation sowie einer chronischen inflammatorischen Reaktion haben dabei ein erhöhtes Risiko für eine akute Exazerbation $[8,10,11]$.

Im Rahmen einer akuten Exazerbation kommt es zu einer akuten inflammatorischen Reaktion (z.B. über Interleukin-8, TNF- $\alpha$, MPO) [12]. Zudem konnte in Untersuchungen der Subgruppe von Patienten mit rezidivierenden akuten Exazerbationen ein erhöhtes Risiko für einen beschleunigten Progress der COPD im Sinne eines funktionellen Verlustes nachgewiesen werden $[13,14]$.

Da die häufigsten Kolonisations-Erreger gleichzeitig auch die führenden mutmaßlichen Erreger der akuten Exazerbation darstellen, liegt die Vermutung nahe, dass es sich um identische Pathogene handelt. Dies ist jedoch nicht immer der Fall. Patienten mit einer akuten Exazerbation können einen neu erworbenen bakteriellen Erreger einer Spezies aufweisen, mit der bereits eine Kolonisation bestand (z.B. Haemophilus influenzae). Die Bildung einer spezifischen Immunantwort gegen neu erworbene Erreger konnte ebenfalls demonstriert werden [12].

Diese Beobachtungen begründen die Sicht, dass bakterielle Erreger bereits als Kolonisationskeime die Progression der COPD be- günstigen und eine ursächliche Rolle in der akuten Exazerbation spielen.

Einschränkend muss jedoch betont werden, dass bakterielle Erreger keineswegs regelmäßig bei akuten Exazerbationen gefunden werden. Vielmehr beträgt die Nachweisrate ca. 50\% [6,15-17]. Die ursächliche Bedeutung der serologisch in ca. 5-10\% nachweisbaren atypischen bakteriellen Erreger (z. B. Chlamydia pneumoniae, Mycoplasma pneumoniae, Legionella spp.) ist ungeklärt [18-25]. Ebenso ist die relative Bedeutung viraler Erreger unzureichend untersucht. Nach neueren Daten sind virale Erreger in bis zu 80\% der Patienten mit akuten Exazerbationen nachweisbar $[11,26]$. Die spezifische Interaktion von viralen und bakteriellen Erregern ist jedoch ebenfalls noch ungeklärt [27].

Aus einer kausalen Rolle bakterieller Erreger in der Pathogenese der akuten Exazerbation folgt schließlich keineswegs zwingend, dass eine antimikrobielle Therapie bronchialer Infektionen auch in einem Maße wirksam ist, das klinisch relevante Vorteile für die Patienten erbringt. Im Gegenteil ist sogar denkbar, dass ein kurzfristiger therapeutischer Gewinn im Sinne einer Verkürzung der Krankheitsdauer langfristig durch die Begünstigung des Erwerbs von Problemkeimen als Folge wiederholter antimikrobieller Therapiezyklen wie Pseudomonas aeruginosa konterkariert wird. Tatsächlich ist die Datenlage zu dieser Frage noch so begrenzt, dass weitere Plazebo-kontrollierte Studien zur Wirksamkeit der antimikrobiellen Therapie unter Einschluss ihrer Langzeiteffekte auf den Verlauf der COPD bzw. die bronchialen Kolonisationserreger erforderlich sind.

\section{Klinische Studien zur antimilkrobiellen Therapie der akuten Exazerbation}

\section{Plazebo-kontrollierte Studien}

Insgesamt liegen 11 Plazebo-kontrollierte Studien sowie eine Metaanalyse zur antimikrobiellen Therapie akuter Exazerbationen der chronischen Bronchitis bzw. COPD vor. Die meisten der 11 Studien (nämlich sechs) sind mehr als 30 Jahre alt und genügen heutigen Qualitätsanforderungen nicht mehr, haben überwiegend Patienten mit chronischer Bronchitis (nicht notwendigerweise COPD) untersucht und haben antimikrobielle Substanzen eingesetzt, die heute nicht mehr primär eingesetzt werden. Methodisch muss darüber hinaus bemerkt werden, dass keine einzige Studie hinsichtlich des Einsatzes von Steroiden kontrolliert ist. Dies wiegt besonders schwer, da Steroiden eine führende Rolle in der Therapie von Patienten mit akuten Exazerbationen zukommt. Darüber hinaus variieren die primären Endpunkte erheblich und entsprechen nicht mehr denen, die aktuell gefordert werden müssen. So berücksichtigt etwa keine der vorliegenden Studien Langzeiteffekte der antimikrobiellen Therapie.

Eine oft zitierte Metaanalyse fußt auf 9 Studien [28], hat zwei neuere Studien nicht eingeschlossen $[29,30]$ und speist das Gewicht ihrer Schlussfolgerung eines kleinen, aber signifikanten Vorteils der antimikrobiellen Therapie gegenüber Plazebo überwiegend aus der bisher größten Studie [31]. Sie hat daher kein zusätzliches Gewicht. 
Tab. 1 Randomisierte Plazebo-kontrollierte Studien zur antimikrobiellen Therapie der akuten Exazerbation der COPD

\begin{tabular}{|c|c|c|c|c|c|}
\hline Studie & Setting & Eingesetzte Substanzen & $\begin{array}{l}\text { Patienten- } \\
\text { zahl }\end{array}$ & $\begin{array}{l}\text { Schweregrad der COPD } \\
\left(F_{1}\right)\end{array}$ & $\begin{array}{l}\text { Effekt der antimikrobiellen } \\
\text { Therapie }\end{array}$ \\
\hline Nicotra u. Mitarb., 1982 & stationär & Tetracyclin & 40 & $\begin{array}{l}0,88 \pm 0,33 \text { und } 0,92 \pm \\
0,54 \mathrm{l} / \mathrm{sec}\end{array}$ & kein Effekt \\
\hline Anthonisen u. Mitarb., 1987 & ambulant & $\begin{array}{l}\text { Amoxicillin, Cotrimoxazol, } \\
\text { Doxycyclin }\end{array}$ & 173 & $33,9 \pm 13,7 \%$ des Solls & Effekt für Typ I \\
\hline Jorgensen u. Mitarb., 1992 & ambulant & Amoxicillin & 278 & keine Messung & kein Effekt \\
\hline
\end{tabular}

Insgesamt verbleiben lediglich fünf Studien, die $<25$ Jahre alt sind [29-33]. Diese finden sich in Tab.1 zusammengefasst. Es muss hervorgehoben werden, dass es nur eine einzige Plazebokontrollierte Studie zu hospitalisierten Patienten gibt (diese umfasst 40 Patienten!) sowie nur eine Studie zu Patienten mit schweren Exazerbationen auf der Intensivstation.

Die Studie von Nicotra u. Mitarb. ist auch nach heutigen Kriterien methodisch valide, umfasst jedoch nur 40 hospitalisierte Patienten. Ein Effekt der antimikrobiellen Therapie wurde nicht gefunden. Die eingesetzte antimikrobielle Substanz war allerdings Tetracyclin. Daher kommt ihr heute keine Relevanz mehr zu [33].

Die klassische Untersuchung von Anthonisen u. Mitarb. ist unverändert die methodisch beste und größte Studie [31]. Ihre Stärke liegt in der Definition von Subgruppen (siehe Tab. 2). Diese wurden zwar bisher nicht validiert; eine aktuelle Studie konnte jedoch belegen, dass eine gelb-grünliche Sputumfarbe tatsächlich ein Prädiktor für das Vorliegen bakterieller Erreger im Sputum war [34]. Die Einschränkungen der Studie ergeben sich aus den Einschlusskriterien (überwiegend schwergradige COPD, mittlere $\mathrm{FEV}_{1}$ von $33,9 \%$ des Solls, ambulant behandelte Exazerbationen), aus der Auswahl der eingesetzten antimikrobiellen Substanzen (Doxycyclin, Cotrimoxazol, Amoxicillin) sowie aus dem Fehlen der Untersuchung von Langzeiteffekten.

Diese Studie wird von manchen Autoren als definitiver Nachweis einer Wirksamkeit der antimikrobiellen Therapie bei akuten Exazerbationen angesehen. Die Ergebnisse müssen jedoch kritisch interpretiert werden. Nur $42 \%$ der Patienten erhielten systemische Steroide. Ein signifikanter Effekt der antimikrobiellen Therapie (definiert als Rückbildung aller Exazerbations-assozi-

Tab. 2 Anthonisen-Kriterien zur Typisierung der akuten Exazerbation der COPD (nach [30])

Typ I: Zunahme von Sputummenge, Purulenz des Sputums und Dyspnoe

Typ II: zwei der drei Kriterien erfüllt

Typ III: eines der drei Kriterien plus Fieber und/oder Symptomen einer oberen Atemwegsinfektion ierten Symptome binnen 21 Tagen) fand sich nur für den Vergleich der Gesamtpopulation ( 68 versus 55\%). Innerhalb der vordefinierten Exazerbationstypen war der Effekt für Typ-I-Exazerbationen mit $63 \%$ versus $43 \%$ am höchsten; für Typ-II- und TypIII-Exazerbationen fanden sich nur geringe bis marginale Unterschiede ( $70 \%$ versus $60 \%$ bzw. 74 versus $70 \%$ ). Andererseits ergab sich hinsichtlich der Verschlechterungen im Verlauf mit Interventionserfordernis mit 10 versus $22 \%$ der Typ-I-Exazerbationen ein Vorteil für die antimikrobiell behandelte Gruppe; der Effekt war bei Typ-II-Exazerbationen deutlich geringer (4 versus $8 \%$ ) und bei Typ-III-Exazerbationen nicht mehr nachweisbar ( $4 \%$ in beiden Gruppen).

Es zeigen sich demnach (verglichen mit ambulant erworbenen Pneumonien) geringe Erfolgsraten bzw. ungewöhnlich hohe Plazebo-Ansprechraten. Diese Ergebnisse sprechen somit allenfalls für einen begrenzten Vorteil der antimikrobiellen Therapie in bestimmten Subgruppen.

Die in der Studie von Jorgensen u. Mitarb. eingeschlossene Patienten-Population entspricht der ambulant behandelten Population mit akuter Exazerbation sehr weitgehend [32]. Ein Effekt der antimikrobiellen Therapie fand sich nicht. Allerdings wurden Patienten mit chronischer Bronchitis nach WHO-Definition (nicht notwendigerweise COPD-Patienten) eingeschlossen.

In die Studie von Sachs u. Mitarb. wurden entsprechend der "dutch hypothesis“ Patienten mit Asthma und COPD-Patienten gleichermaßen eingeschlossen [29]. Auch diese Studie fand keinen Effekt der antimikrobiellen Therapie. Die Beeinträchtigung der Lungenfunktion war jedoch sehr gering bis marginal. Schließlich wurden Amoxicillin und Cotrimoxazol eingesetzt. Die Aussagekraft dieser Studie bleibt daher limitiert.

Die einzige Studie zu schweren Exazerbationen auf der Intensivstation hat Patienten mit schwerer COPD untersucht (mittlere $\mathrm{FEV}_{1} 0,74-0,79$ l) [30]. Sie weist eine Fülle von Limitationen auf. Kein Patient erhielt Steroide; die Rate an gescheiterten nichtinvasiven Beatmungsversuchen war ungewöhnlich hoch; die eingesetzte Substanz Ofloxacin ist aufgrund der nicht ausreichenden Pneumokokken- und Pseudomonas-Aktivität in dieser Population fragwürdig; wahrscheinlich wurde die beobachtete Re- 
duktion der Letalität überwiegend aufgrund einer Reduktion der ungewöhnlich hohen Rate an früher („early onset“) nosokomialer Pneumonie erzielt; schließlich wies die Population eine ungewöhnlich niedrige Rate an Pseudomonas aeruginosa auf, was die Repräsentativität der untersuchten Population beeinträchtigt.

\section{Hinweise zur Wirksamkeit antimikrobieller Therapien aus anderen Studien}

Diese Hinweise sind naturgemäß äußerst divergent, ergeben jedoch wichtige Anfragen an Validität und Design der bisherigen Ergebnisse Plazebo-kontrollierter Studien. Hier einige wichtige Beispiele:

Im Rahmen einer Studie von 50 Patienten mit schwerer, beatmungspflichtiger akuter Exazerbation wurden bronchoskopisch über die geschützte Bürste (PSB) in 50\% der Fälle bakterielle Erreger in hoher Keimzahl isoliert. Eine antimikrobielle Therapie erfolgte nicht, dennoch unterschied sich der Ausgang dieser Patienten nicht von denen ohne Nachweis bakterieller Erreger [16].

Das Vorliegen häufig rezidivierender Exazerbationen ( $\geq 4 / \mathrm{Jahr}$ ) sowie einer kardialen Komorbidität wurde als bester Prädiktor für ein Therapieversagen der antimikrobiellen Therapie identifiziert [35]. In einer anderen Arbeit wurden in multivariater Analyse lediglich das Bestehen einer Sauerstoff-Langzeittherapie (LTOT) bzw. die Frequenz akuter Exazerbationen als Prädiktoren eines Therapieversagens identifiziert; das Alter, die Komorbidität sowie die Auswahl mikrobieller Therapie waren jeweils nicht prädiktiv [36].

Es konnte gezeigt werden, dass die Rezidivrate von Patienten mit akuter Exazerbation geringer war, wenn diese mit Antibiotika behandelt worden waren. Dies galt allerdings nicht für alle antimikrobiellen Substanzen. Unter Amoxicillin war die Rezidivrate am höchsten [37].

In der bisher einzigen Therapiestudie, in der die antimikrobielle Therapie für die Gabe von (inhalativen und systemischen) Steroiden kontrolliert war, konnte ein überlegener Effekt von Moxifloxacin gegenüber Vergleichssubstanzen in der Gruppe ohne gleichzeitige Steroidtherapie nachgewiesen werden [38]. Diese Beobachtung unterstreicht, dass heute Aussagen zur Wirksamkeit antimikrobieller Therapien nur zulässig sind, wenn diese für die Gabe von Steroiden kontrolliert sind.

\section{Indikationen für eine antimikrobielle Therapie}

Die Ergebnisse lassen sich in aller Vorläufigkeit wie folgt zusammenfassen:

- Patienten mit einer leichtgradigen COPD (überwiegend, aber nicht immer gleichbedeutend mit leichtgradiger Exazerbation) profitieren von einer antimikrobiellen Therapie nicht oder nur marginal. Dies gilt zumindest für die in den Studien eingesetzten antimikrobiellen Substanzen.

- Patienten mit einer mittel- bis schwergradigen COPD (nicht notwendig identisch mit mittel- bis schwergradiger Exazerbation) profitieren in begrenztem Umfang von einer antimikrobiellen Therapie in folgenden Subgruppen:
- ambulant behandelte Patienten mit Typ-I-Exazerbation,

- beatmungspflichtige akute Exazerbationen,

- keine verwertbaren Daten sind verfügbar für

- stationär behandelte Patienten (außerhalb der Intensivstation),

- Patienten mit häufig rezidivierenden akuten Exazerbationen.

Auf diesem Hintergrund lassen sich folgende mögliche Indikationen für eine antimikrobielle Therapie versuchsweise formulieren:

- Patienten mit mittelschwerer bis schwerer COPD, die einen Anthonisen-Typ I aufweisen.

- Patienten mit schwerer akuter Exazerbation, die eine respiratorische Unterstützung brauchen (NIV oder maschinelle Beatmung).

Obwohl keine verwertbaren Daten für Patienten aller Schweregrade der Exazerbation und/oder COPD mit rezidivierenden akuten Exazerbationen ( $\geq 4 / \mathrm{Jahr}$ ) vorliegen, handelt es sich zweifellos um eine durch ihre Morbidität besonders belastete Gruppe; eine antimikrobielle Therapie lässt sich daher auch in dieser Patientengruppe rechtfertigen. Wahrscheinlich sollte diese Gruppe jedoch nur gezielt nach Kulturergebnis und Antibiogramm und nicht kalkuliert behandelt werden.

Auch diese restriktiv gehaltene Indikationsliste geht noch von der Vermutung aus, dass eine antimikrobielle Therapie in den genannten Gruppen kurz- und langfristig eher Vorteile als Nachteile erbringt; wie oben ausgeführt, ist dies jedoch keinesfalls gesichert.

Auswahl des Behandlungsorts sowie Differenzialindikation zur antimilkrobiellen Therapie entsprechend dem Schweregrad der akuten Exazerbation

In den verfügbaren Leitlinien von Fachgesellschaften fußen die differenzialtherapeutischen Kriterien wesentlich auf Schweregraden der COPD. So wird unterschieden zwischen Patienten mit leichtgradiger COPD, die orale Basis-Präparate erhalten und solchen mit mittel- bis schwerergradiger COPD, die mit Präparaten mit breiterem Spektrum behandelt werden müssen. Stets wird auch auf diesem Boden eine kalkulierte antimikrobielle Therapie entsprechend den Empfehlungen bei Pneumonie abgeleitet.

Dies ist in mehrfacher Hinsicht fragwürdig. Zum einen ist der Zusammenhang der Schweregrade von COPD und akuter Exazerbation im Einzelfall unsicher; so kann ein Patient mit leichtgradiger COPD durchaus eine schwergradige Exazerbation erleben und umgekehrt (siehe Abb.1). Darüber hinaus ergibt sich das praktische Problem, dass nicht für alle Patienten mit akuter Exazerbation der Schweregrad der COPD bekannt ist. Zum anderen stellt sich die Frage, inwiefern Schweregrade der COPD überhaupt relevante differenzialtherapeutische Kriterien für Patienten mit akuter Exazerbation darstellen können. Im Unterschied zur Pneumonie sind Patienten mit akuter Exazerbation nie durch ein oxygenatorisches Versagen oder eine schwere Sepsis bedroht, sondern einzig durch ein ventilatorisches Versagen (siehe 


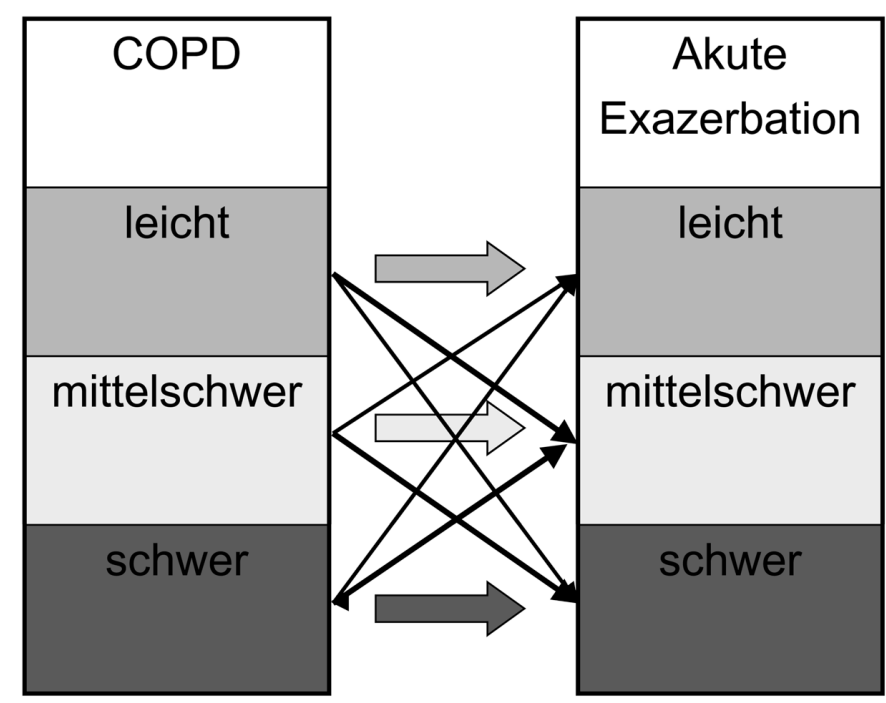

Abb. 1 Zusammenhang der Schweregrade der COPD mit den Schweregraden der akuten Exazerbation. Es besteht keine eindeutige Assoziation zwischen den Schweregraden der COPD und der akuten Exzerbation.

Abb. 2). Während jedoch die Bedeutung der frühzeitigen adäquaten antimikrobiellen Therapie der schweren Sepsis hinreichend belegt ist, ist aktuell unbekannt, ob durch eine antimikrobielle Therapie ein ventilatorisches Versagen beeinflusst werden kann. Ob eine frühzeitige kalkulierte Therapie überhaupt in jedem Fall erforderlich ist oder auch bis zu einem Erregernachweis abgewartet und gezielt behandelt werden kann, ist entsprechend ebenfalls nicht untersucht.

In praktischer Hinsicht sind analog der Behandlung von Patienten mit Pneumonien zunächst Kriterien für die Auswahl des Behandlungsorts (ambulant/stationär/Intensivstation) relevant. Diese müssen sich aus dem klinischen Zustand des Patienten ableiten. Entsprechend ist eine Orientierung des Behandlungskonzepts am Schweregrad der akuten Exazerbation (nicht primär der COPD!) zu fordern.

Kriterien für einen Schweregrad der akuten Exazerbation sind allerdings bislang nicht systematisch evaluiert worden. Wieder analog der Pneumonie lassen sich Schweregradkriterien am ehesten aus unabhängigen Prädiktoren der Letalität der akuten Exazerbation ableiten. Hier zeigt sich, dass die ventilatorische Insuffizienz sowie die Komorbidität die entscheidenden Faktoren darstellen $[39,40]$. Diese prognostisch relevanten Kriterien lassen sich wie folgt pragmatisch formulieren (siehe Abb. 3):

- Leichtgradige Exazerbation, ambulant behandelbar:

- Allgemeinzustand gut bis ausreichend,

- keine Notwendigkeit einer Sauerstoffgabe bzw. respiratorischen Unterstützung,

- kein erhöhtes Risiko der Dekompensation einer extrapulmonalen Komorbidität.

- Mittelschwere Exazerbation, stationär behandlungspflichtig:

- Allgemeinzustand erheblich beeinträchtigt,

- Notwendigkeit der Sauerstoffgabe und/oder,

- Risiko der Dekompensation oder manifeste leicht- bis mittelgradige Dekompensation einer extrapulmonalen Erkrankung.

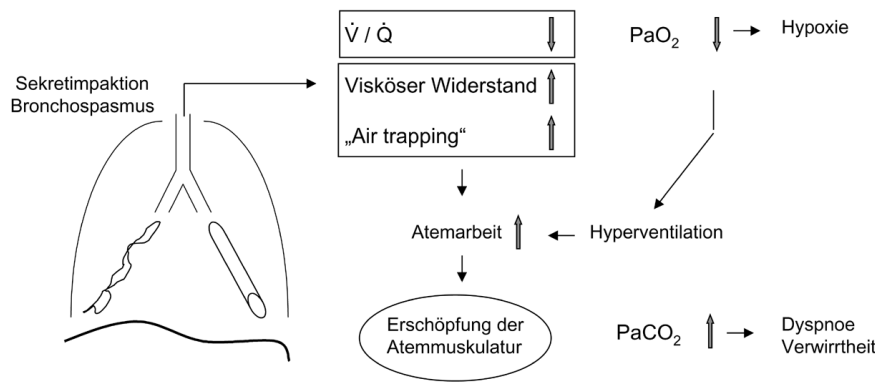

Abb. 2 Pathophysiologie der schweren akuten Exazerbation der COPD. Im Unterschied zur Pneumonie steht die ventilatorische Insuffizienz im Vordergrund.

\begin{tabular}{|l|c|c|c|c|c|}
\hline $\begin{array}{l}\text { Schwere- } \\
\text { grad }\end{array}$ & Setting & Komorbidität & $\begin{array}{c}\text { Allgemein- } \\
\text { zustand }\end{array}$ & $\begin{array}{c}\mathrm{O}_{2} \text {-Subs- } \\
\text { titution } \\
\text { erforderlich }\end{array}$ & $\begin{array}{c}\text { Beatmung } \\
\text { erforderlich }\end{array}$ \\
\hline leicht & ambulant & stabil & $\begin{array}{c}\text { gut - } \\
\text { ausreichend }\end{array}$ & - & - \\
\hline $\begin{array}{l}\text { mittel- } \\
\text { schwer }\end{array}$ & stationär & $\begin{array}{c}\text { stabil } \\
\text { instabil }\end{array}$ & $\begin{array}{c}\text { beein- } \\
\text { trächtigt }\end{array}$ & + & + \\
\hline schwer & $\begin{array}{c}\text { intermediate } \\
\text { care oder } \\
\text { ICU }\end{array}$ & $\begin{array}{c}\text { stabil } \\
\text { instabil }\end{array}$ & $\begin{array}{c}\text { beein- } \\
\text { trächtigt } \\
\text { oder } \\
\text { schlecht }\end{array}$ & + & + \\
\hline
\end{tabular}

Abb. 3 Schweregrade der akuten Exazerbation und Kriterien für eine Hospitalisation.

- Schwere Exazerbation, behandlungspflichtig auf intermediate care- oder Intensivstation:

- Notwendigkeit der respiratorischen Unterstützung.

- schwergradige Dekompensation einer extrapulmonalen Erkrankung.

Patienten mit einer bestehenden Sauerstoff-Langzeittherapie müssen gesondert betrachtet werden, da bei diesen das Kriterium der „Sauerstoffpflichtigkeit“ als solches nicht verwertet werden kann. In dieser Gruppe muss dieses Kriterium modifiziert werden in „Steigerung des Sauerstoff-Bedarfs“.

Die genannten Schweregrad-Kriterien werden ihrerseits durch folgende einfache klinische Parameter reflektiert:

- Leichtgradige Exazerbation,

- keine schwere Dyspnoe, keine schwere Komorbidität.

- Mittelschwere Exazerbation

- schwere Dyspnoe,

- schwere Komorbidität.

- Schwergradige Exazerbation:

- Zeichen der ventilatorischen Erschöpfung (Hyper- oder Hypoventilation, i.e. Atemfrequenz $>30$ oder $<12 / \mathrm{min}$, paradoxe Atmung),

- Respiratorische Azidose $(\mathrm{pH}<7,3$ ) (ggf. trotz Sauerstoffgabe),

- Bewusstseinstrübung,

- jegliches Zeichen einer schweren kardialen Dekompensation. 


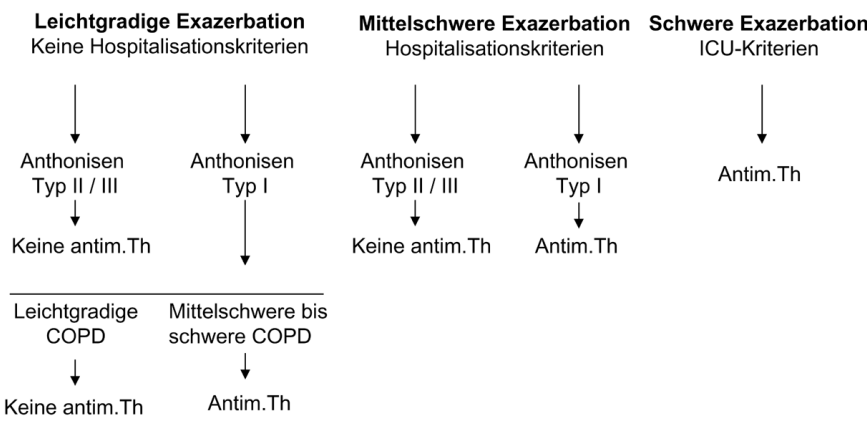

Abb. 4 Algorithmus der Indikationen zur antimikrobiellen Therapie der akuten Exazerbation der COPD

antim. Therapie $=$ antimikrobielle Therapie.

Selbstverständlich entscheiden über den Schweregrad hinaus auch soziale Kriterien darüber, ob eine stationäre Aufnahme erforderlich wird (z. B. Fähigkeit der Selbstversorgung).

Diese Kriterien zur Hospitalisation bzw. Aufnahme auf einer intermediate care- bzw. Intensivstation entsprechen denen, die im gemeinsamen Dokument der ATS und ERS vorgeschlagen wurden [1]. Wie im Rahmen einer akuten Exazerbation der Allgemeinzustand bzw. das Ausmaß der Komorbidität objektiviert werden kann, ist aktuell nicht untersucht und muss daher aktuell allein dem klinischen Urteil überlassen bleiben.

Schließlich können in diese Schweregrade die Indikationen für eine antimikrobielle Therapie eingefügt werden (siehe Abb.4). Demnach erhalten eine solche Therapie Patienten mit einer akuten Exazerbation vom Anthonisen-Typ I bei leichtgradiger Exazerbation nur dann, wenn eine mittelschwere bis schwere COPD vorliegt sowie stets bei mittelschwerer Exazerbation. Patienten mit schwergradiger akuter Exazerbation erhalten immer eine antimikrobielle Therapie.

Ein Problem aus klinischer Erfahrung, das noch nicht systematisch untersucht worden ist, besteht darin, dass einige Patienten nicht in der Lage sind, Sputum zu produzieren, gleichwohl eine massive Sekretretention besteht. In der Praxis sollten diese Patienten als Typ-I-Exazerbationen klassifiziert werden. Zusätzlich zur antimikrobiellen Therapie ist hier die bronchoskopische Bronchialtoilette (ggf. wiederholt) anzuwenden.

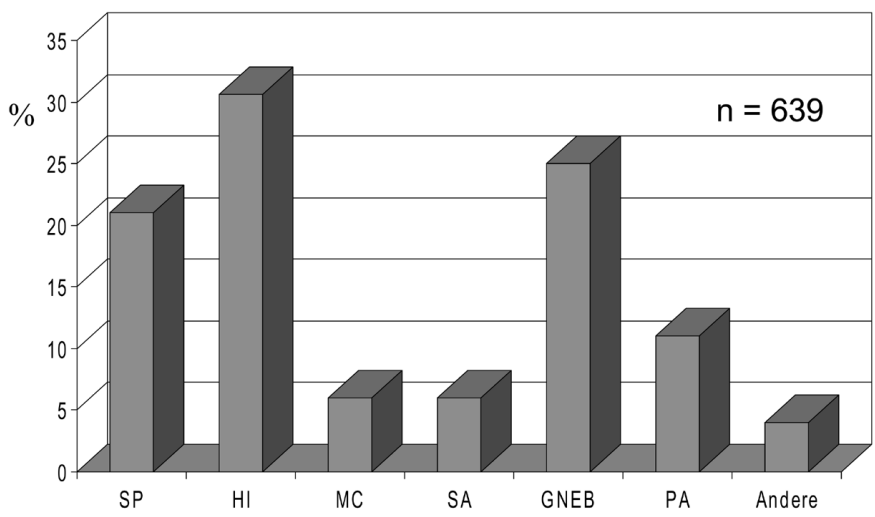

Abb. 5 Erregerspektrum der akuten Exazerbation der COPD. $\mathrm{SP}=$ Streptococcus pneumoniae; $\mathrm{HI}=$ Haemophilus influenzae; $\mathrm{MC}=$ Moraxella catarrhalis; $\mathrm{SA}=$ Staphylococcus aureus; $\mathrm{GNEB}=\mathrm{Gram}$ negative Enterobakterien; $\mathrm{PA}=$ Pseudomonas aeruginosa [41].
Kriterien der antimikrobiellen Substanzauswahl

Wichtige Faktoren im Rahmen der antimikrobiellen Substanzauswahl umfassen das Erreger- und Resistenzspektrum, Vergleichsstudien antimikrobieller Substanzen sowie Strategien zur Limitation des Selektionsdrucks.

\section{Erregerspektrum}

Haemophilus influenzae und Streptococcus pneumoniae sind die führenden bakteriellen Erreger der akuten Exazerbation der COPD (Abb. 5) [41]. Moraxella catarrhalis wird von manchen Autoren als dritthäufigster Erreger genannt; die begrenzte Inzidenz dieses Erregers rechtfertigt jedoch keine prominente Nennung. Aus mehreren Studien ist bekannt, dass sich das bakterielle Erregerspektrum mit zunehmendem Schweregrad der COPD im Sinne einer Häufung von Gram-negativen Enterobakterien und Pseudomonas aeruginosa verändert $[15-17,42,43]$. Dieser Wandel im Erregerspektrum erklärt sich in erster Linie auf dem Hintergrund häufiger Hospitalisationen bzw. wiederholter antimikrobieller Therapiezyklen (und begründet die grundlegende Skepsis gegenüber den Langzeitfolgen der antimikrobiellen Therapie). Die Bedeutung „atypischer“ Erreger ist unbekannt; aktuell wird eine Berücksichtigung dieser Erreger im antimikrobiellen Spektrum nicht für erforderlich gehalten. Studien zu Neuraminidase-Hemmern bei Patienten mit akuten Exazerbationen mit Verdacht auf Influenzavirus liegen nicht vor. Ihr Einsatz bei ausgewählten Patienten mit schwerer COPD und durch Schnelltest gesichertem Influenzavirusnachweis ist zu erwägen.

Ein Zusammenhang von Erregerspektrum und Schweregrad der Exazerbation ist nicht gesichert. Da eine COPD mit höherem Schweregrad jedoch mit höherer Wahrscheinlichkeit auch eine schwergradige akute Exazerbation nach sich zieht, ist bei einer schwergradigen akuten Exazerbation die Wahrscheinlichkeit eines veränderten Erregerspektrums (i.e. einer höheren Inzidenz von Gram-negativen Enterobakterien und Pseudomonas aeruginosa) entsprechend erhöht.

\section{Resistenzspektrum}

Das aktuelle Resistenzspektrum in Deutschland ist dadurch gekennzeichnet, dass die Inzidenz $\beta$-Laktamase-bildender Haemophilus influenzae ca. $5-15 \%$ beträgt, die Inzidenz Penicillin-resistenter Pneumokokken noch sehr gering ist $(<5 \%)$ und die Inzidenz Makrolid-resistenter Pneumokokken jedoch bereits an 20\% heranreicht (Tab. 3) [44]. In welchem Umfang mikrobielle Resistenzen in der Therapie der akuten Exazerbation überhaupt relevant sind, ist ungeklärt. Immerhin konnten in einer Vergleichsstudie von Moxifloxacin mit anderen Basissubstanzen Unterschiede in den Eradikationsraten von Haemophilus influenzae beobachtet werden, was wahrscheinlich auf die Wirkschwäche von Makroliden gegenüber diesem Erreger zurückzuführen ist [38].

\section{Vergleichsstudien antimikrobieller Substanzen}

Eine konsistente Überlegenheit einer Substanz über andere Substanzen konnte bisher nicht belegt werden. Allerdings liegen bisher überwiegend nur Zulassungsstudien für die Indikation akute Exazerbation vor, deren Qualität in vielfacher Hinsicht (PatientenAuswahl und Charakterisierung, Wahl der Endpunkte, Stratifizierungen für Störgrößen, Einfluss auf Langzeitverlauf der COPD) un- 
befriedigend bleibt [45]. In einer der ganz wenigen Studien mit qualitativ anspruchsvollem Design konnte gezeigt werden, dass Moxifloxacin gegenüber einer Therapiestrategie bestehend aus je nach klinischem Ermessen - Amoxicillin, Cefuroxim-Axetil oder Clarithromycin in allen vordefinierten Endpunkten um ca. $10 \%$ überlegen war [38]. Darüber hinaus stellen Fluorchinolone wie Ciprofloxacin und Moxifloxacin die bisher einzigen Substanzen dar, die das Potenzial aufweisen, die Dauer bis zur nächsten Exazerbation zu verlängern $[37,46,47]$. Dieser Effekt konnte für Levofloxacin in einer Population mit überwiegend leichtgradiger akuter Exazerbation nicht nachgewiesen werden [48].

\section{Limitation des Selektionsdrucks}

Patienten mit COPD werden voraussehbar wiederholten antimikrobiellen Therapiezyklen unterworfen werden müssen. Daher sind neben dem Schweregrad und dem Erreger- und Resistenzspektrum auch die Limitierung der Entwicklung von mikrobiellen Resistenzen wichtige Kriterien für die Selektion der antimikrobiellen Therapie. Nach neuesten Daten scheint eine intraindividuelle Rotationsstrategie in der Lage, zumindest Resistenzentwicklungen von Streptococcus pneumoniae vorzubeugen. Es kommt daher darauf an, innerhalb einer Reihe von gleichwertig adäquaten antimikrobiellen Substanzen zu rotieren, und zwar auch dann, wenn der letzte Therapiezyklus mit einer bestimmten Substanz erfolgreich durchgeführt werden konnte. Das Zeitintervall, in dem von einem relevanten Einfluss einer vorhergehenden antimikrobiellen Therapie auf die Entwicklung einer Resistenz ausgegangen werden muss, beträgt 3-6 Monate [49].

\section{Substanzklassen und Substanzen}

Grundsätzlich adäquate Substanzen bzw. Substanzklassen für die orale Basistherapie sind (siehe Tab. 3 u. 4):

- Penicilline (Amoxicillin bzw. Amoxicillin-Clavulansäure/Sultamicillin),

- Tetracycline (Doxycyclin),

- Fluorchinolone (Moxifloxacin, Levofloxacin),

- Makrolide (Clarithromycin, Azithromycin),

- Ketolide (Telithromycin).

Die Entscheidung über den Einschluss eines $\beta$-Laktamasehemmers sollte entsprechend den regionalen Resistenzdaten erfolgen. Beträgt die Rate $\beta$-Laktamase-bildender Haemophilus influenzae z.B. > 10\% oder ist diese unbekannt, kann dieser hinzugefügt werden. Tetracycline sollten nur zum Einsatz kommen, wenn die regionale Resistenzrate gegenüber Streptococcus pneumoniae $<10 \%$ beträgt. Unter den Fluorchinolonen sind Moxifloxacin und Levofloxacin aufgrund der hervorragenden Wirksamkeit gegen Haemophilus influenzae und Streptococcus pneumoniae die Basis-Substanzen. Ciprofloxacin ist nicht ausreichend wirksam gegen Streptococcus pneumoniae und sollte daher ausschließlich im Rahmen der gezielten Therapie von Pseudomonas aeruginosa zum Einsatz kommen.

Makrolide und Ketolide haben in vitro eine relativ schwache Wirkung gegenüber Haemophilus influenzae. Unter den Makroliden haben Clarithromycin und Azithromycin die relativ beste Wirkung gegenüber diesem Erreger. Möglicherweise ist ihre Wirksamkeit aufgrund ihrer intrazellulären Wirksamkeit sowie immunmodulatorischer Eigenschaften jedoch in vivo besser als dies nach in vitro Daten nahegelegt wird. Der Vorteil der Ketolide liegt in seiner Wirksamkeit auch gegen Makrolid-resistente Streptococcus pneumoniae.

Aufgrund der exzellenten Wirksamkeit und Pharmakokinetik, des vorteilhaften Effekts auf die Dauer der Exazerbationsintervalle sowie eines gesicherten Vorteils von ca. $10 \%$ gegenüber den anderen Standardsubstanzen gehört Moxifloxacin zu den am aktivsten und am besten dokumentierten Substanzen in der Therapie von Patienten mit akuten Exazerbationen. Neben seinem Einsatz im Rahmen eines „Rotationskonzepts“ besteht ein besonderer potenzieller Vorteil bei Patienten, die eine schwere akute Exazerbation oder die häufige Exazerbations-Rezidive erleben.

Orale Cephalosporine haben gegenüber Amoxicillin plus $\beta$-Laktamasehemmer keine Vorteile. Aufgrund ihrer vergleichsweise unsicheren Resorption werden sie nicht empfohlen.

Für die intravenöse Therapie stehen zur Verfügung:

- Penicilline (Ampicillin bzw. Amoxicillin-Clavulansäure/Ampicillin-Sulbactam; Piperacillin-Tazobactam),

- Cephalosporine (Cefuroxim, Ceftriaxon, Cefotaxim, Ceftazidim),

- Makrolide (Clarithromycin),

- Fluorchinolone (Moxifloxacin, Levofloxacin, Ciprofloxacin).

Unter den Penicillinen und Cephalosporinen müssen Pseudomonas-aktive von -inaktiven Substanzen unterschieden werden. Für die bislang häufig empfohlene Kombinationstherapie der akuten Exazerbation durch Pseudomonas aeruginosa mit $\beta$-Laktamen mit Aminoglykosiden sind keine Daten verfügbar. Mehrere Metaanalysen zu einer entsprechenden Kombinationstherapie bei Patienten mit Sepsis haben keinen positiven Effekt hinsichtlich Ansprechen, Letalität sowie Resistenzverhütung unter Therapie gezeigt, sondern lediglich eine Erhöhung der Toxizität [50,51]. Entsprechend sollten Aminoglykoside daher in der Regel nicht gegeben werden. Die Kombination von $\beta$-Laktamen mit Fluorchinolonen bei akuten Exazerbationen ist bislang ebenfalls nicht untersucht.

\section{Antimikrobielle Therapieoptionen}

Zur Therapie der akuten Exazerbation ergeben sich folgende Optionen:

Bei leichtgradigen akuten Exazerbationen mit Indikation für eine antimikrobielle Therapie sollte eine Rotation zwischen den oralen Basissubstanzen erfolgen. Die Therapiedauer beträgt - je nach eingesetzter Substanz - 3 bis 7 Tage.

Bei mittelschweren akuten Exazerbationen sollte eine Rotation zwischen Penicillinen (Amoxicillin bzw. Amoxicillin-Clavulansäure/Sultamicillin) und Fluorchinolonen (Moxifloxacin, Levofloxacin) durchgeführt werden. Eine Therapiedauer von 7 Tagen sollte ausreichend sein.

Es gibt keine Evidenz dafür, dass eine kalkulierte Therapie von Pseudomonas aeruginosa vor einem entsprechenden Erreger- 
Tab. 3 Spektrum antimikrobieller Substanzen gegenüber führenden bzw. möglichen Erregern der akuten Exazerbation der COPD. Die Bedeutung mikrobieller Resistenzen für den Therapieerfolg bei akuter Exazerbation der COPD ist im Einzelnen unbekannt. Dennoch liegt es nahe, das Spektrum der infrage kommenden Substanzen bei der Substanzauswahl zu berücksichtigen

\begin{tabular}{|c|c|c|c|c|c|c|}
\hline $\begin{array}{l}\text { Anti-mikrobielle } \\
\text { Substanz }\end{array}$ & H. influenzae & S. Pneumoniae & $\begin{array}{l}\text { Resistente } \\
\text { S. pneumoniae }\end{array}$ & M. catarrhalis & Atypische & P. aeruginosa \\
\hline \multicolumn{7}{|l|}{ Penicilline } \\
\hline Amoxicillin & \pm & + & $P+, M+$ & - & - & - \\
\hline $\begin{array}{l}\text { Amoxicillin } \\
+\beta-\mathrm{LI}\end{array}$ & + & + & $P+, M+$ & + & - & - \\
\hline Lorafem & + & + & $\mathrm{P} \pm, \mathrm{M}+$ & + & - & - \\
\hline Cefixim & + & + & $P \pm, M+$ & + & - & - \\
\hline \multicolumn{7}{|l|}{ Makrolide } \\
\hline Clarithromycin & \pm & \pm & $\mathrm{P} \pm, \mathrm{M}-$ & + & + & - \\
\hline Roxithromycin & \pm & \pm & $\mathrm{P} \pm, \mathrm{M}-$ & + & + & - \\
\hline Azithromycin & \pm & \pm & $\mathrm{P} \pm, \mathrm{M}-$ & + & + & - \\
\hline \multicolumn{7}{|l|}{ Ketolide } \\
\hline Doxycyclin & + & \pm & $P+/ M+$ & + & + & - \\
\hline \multicolumn{7}{|l|}{ Fluorchinolone } \\
\hline Moxifloxacin & + & + & $P+/ M+$ & + & + & - \\
\hline Levofloxacin & + & + & $P+/ M+$ & + & + & + \\
\hline Ciprofloxacin & + & - & - & + & + & + \\
\hline
\end{tabular}

+ = Wirksamkeit regelmäßig gegeben; \pm = Wirksamkeit abhängig vom Isolat; - = Wirksamkeit regelmäßig nicht gegeben; P + = Wirksamkeit gegen Penicillin-resistente Pneumokokken; P - = keine Wirksamkeit gegen Penicillin-resistente Pneumokokken; M + = Wirksamkeit gegen Makrolid-resistente Pneumokokken; $\mathrm{M}-=$ keine Wirksamkeit gegen Makrolid-resistente Pneumokokken

nachweis erforderlich ist. Eine initiale kalkulierte antipseudomonale Therapie kann daher auf Patienten mit schwerer Exazerbation beschränkt bleiben. Allerdings sollten hospitalisierte Patienten eine Sputumuntersuchung erhalten, um ggf. die Therapie entsprechend gezielt umstellen zu können.

Patienten mit leicht- bis mittelschwerer Exazerbation und Nachweis von Pseudomonas aeruginosa sollten Levofloxacin oder Ciprofloxacin oral erhalten, sofern diese Substanzen als sensibel getestet worden sind.

Schwergradige akute Exazerbationen sollten intravenös behandelt werden. Infrage kommen für die Initialtherapie:

- Penicilline (Piperacillin-Tazobactam),

- Cephalosporine (Ceftazidim, Cefepim),

- Fluorchinolone (Levofloxacin, Ciprofloxacin).

Fluorchinolone können als einzige Substanzen in Form einer Sequenzialtherapie verabreicht werden. Carbapeneme (Imipenem/ Cilastatin, Meropenem) sollten nur in der gezielten Therapie (als Reservesubstanzen) zum Einsatz kommen. Die Dauer der Therapie sollte auf 8 Tage beschränkt bleiben.

Substanzklassen und Dosierungen finden sich in Tab. 3 u. 4 zusammengefasst.

\section{Evaluation des Therapieansprechens}

Kriterien des Ansprechens sind unzureichend definiert. Theoretisch lassen sich symptombezogene und lungenfunktionelle Erfolgskriterien definieren. Die Assoziation von beiden ist begrenzt.

Über das Zeitfenster, innerhalb dessen ein Therapieansprechen zu erwarten ist, gibt es keine Daten. Analog der Pneumonie wird ein solches Ansprechen in ca. 48 - $72 \mathrm{~h}$ zu erwarten sein.

- Zeichen des klinischen (symptombezogenen) Ansprechens umfassen:

- Rückgang der Dyspnoe,

- Rückgang der Sputummenge,

- Aufklaren der Sputumfarbe,

- Besserung der respiratorischen Azidose,

- Besserung des mentalen Zustands,

- Stabilisierung der komorbiden Dekompensation.

Ein signifikanter Anteil von Patienten weist nur eine inkomplette Remission der Exazerbations-Symptome auf. Der therapeutische Umgang mit diesen Patienten ist nicht etabliert. In jedem Fall sollte eine ungezielte protrahierte Gabe antimikrobieller Substanzen (über maximal 7-8 Tage hinaus) vermieden werden, da die Wahrscheinlichkeit einer Selektion resistenter Erreger bzw. (bei Pseudomonas aeruginosa) auch einer Resistenzentwicklung unter Therapie nach diesem Zeitraum deutlich wächst. 
Tab. 4 Antimikrobielle Substanzen zur Therapie der akuten Exazerbation der COPD

\begin{tabular}{|c|c|c|}
\hline Antimikrobielle Substanz & $\begin{array}{l}\text { Verfügbarkeit } \\
\text { oral/i. v. }\end{array}$ & Dosierung \\
\hline \multicolumn{3}{|l|}{$\beta$-Lactame } \\
\hline Amoxicillin & oral & $3 \times 1 \mathrm{~g}$ \\
\hline Amoxicillin/Clavulansäure & oral/i. v. & $\begin{array}{l}2 \times 875 \mathrm{mg} \text { oral } \\
3 \times 2,2 \mathrm{~g} \text { i. } \mathrm{v} .\end{array}$ \\
\hline Sultamicillin & oral & $2 \times 750 \mathrm{mg}$ \\
\hline Piperacillin/Tazobactam & i.v. & $3 \times 4,5 \mathrm{~g}$ \\
\hline \multicolumn{3}{|l|}{ Cephalosporine } \\
\hline Cefuroxim & i.v. & $3 \times 1,5 \mathrm{~g}$ i.v \\
\hline Ceftriaxon & i.v. & $1 \times 1-2 g$ \\
\hline Cefotaxim & i.v. & $3 \times 2 g$ \\
\hline Ceftazidim & i.v. & $3 \times 2 g$ \\
\hline Cefepim & i.v. & $2 \times 2 g$ \\
\hline \multicolumn{3}{|l|}{ Makrolide } \\
\hline Clarithromycin & oral/i.v. & $2 \times 500 \mathrm{mg}$ \\
\hline Azithromycin & oral & $\begin{array}{l}1 \times 500 \mathrm{mg} \text { Tag } 1 \\
1 \times 250 \mathrm{mg} \text { Tage } 2-5 \\
\text { oder } \\
1 \times 500 \mathrm{mg} \text { Tage } 2-3\end{array}$ \\
\hline \multicolumn{3}{|l|}{ Ketolide } \\
\hline Telithromycin & oral & $1 \times 800 \mathrm{mg}$ \\
\hline \multicolumn{3}{|l|}{ Tetracycline } \\
\hline Doxycyclin & oral & $2 \times 100 \mathrm{mg}$ \\
\hline \multicolumn{3}{|l|}{ Fluorchinolone } \\
\hline Ciprofloxacin & oral/i. v. & $\begin{array}{l}2 \times 500-750 \mathrm{mg} \text { oral } \\
2-3 \times 400 \mathrm{mg} \text { i. } \mathrm{v} .\end{array}$ \\
\hline Levofloxacin & oral/i. v. & $1 \times 500 \mathrm{mg}$ \\
\hline Moxifloxacin & oral/i.v. & $1 \times 400 \mathrm{mg}$ \\
\hline
\end{tabular}

\section{Therapieversagen}

Ein Therapieversagen äußert sich in einer persistierenden Symptomatik trotz adäquater Therapie von mindestens 48 - 72 h Dauer. Zum Vorgehen im Rahmen eines Therapieversagens liegen keine Studien vor.

Entsprechend klinischer Erfahrung wird die Erwägung folgender Untersuchungen empfohlen:

- Wiederholte Bestimmung von Entzündungsparametern (Blutbild, CRP),

- Wiederholte Sputumuntersuchung,

- Bronchoskopie zur Gewinnung respiratorischer Sekrete (und zur Bronchialtoilette bei Sekretretention),

- Echokardiographie zum Ausschluss Linksherzdekompensation,

- Röntgen-Thorax zum Ausschluss Pneumonie (primär ambulant erworben bzw. nosokomial),

- Angio-CT des Thorax zum Ausschluss Lungenembolien.

\section{Fazit für die Praxis}

Jedes Konzept der antimikrobiellen Therapie der akuten Exazerbation der COPD weist aufgrund der limitierten Datenlage einen induktiven und tentativen Charakter auf. Dies verpflichtet den behandelnden Pneumologen zu einem selektiven Einsatz antimikrobieller Substanzen. Viel ist schon gewonnen, wenn Patienten mit leichtgradiger akuter Exazerbation und leichtgradiger COPD in der Regel keine antimikrobielle Therapie erhalten. Das Konzept der intraindividuellen Rotation scheint geeignet zu sein, den Selektionsdruck zu reduzieren. Zukünftige Studien werden zu klären haben, welche Patienten tatsächlich am meisten von einer antimikrobiellen Therapie profitieren.

\section{Literatur}

${ }^{1}$ Celli BR, MacNee W, ATS/ERS Task Force. Standards for the diagnosis and treatment of patients with COPD: a summary of the ATS/ERS position paper. Eur Respir J 2004; 23: 932 - 946

${ }^{2}$ Guidelines for the diagnosis and treatment chronic obstructive bronchitis and pulmonary emphysema issued by Deutsche Atemwegsliga and Deutsche Gesellschaft für Pneumologie. Pneumologie 2002; 56: $704-738$

${ }^{3}$ Woodhead M, Blasi F, Ewig S et al. Guidelines for the management of adult lower respiratory tract infections. Eur Respir J 2005; 26 : $1138-1180$

${ }^{4}$ Höffken G, Lorenz L, Kern W et al. S3-Leitlinie zu ambulant erworbener Pneumonie und tiefen Atemwegsinfektionen. Pneumologie 2005; 59: $612-664$

${ }^{5}$ Cabello H, Torres A, Celis R et al. Bacterial colonization of distal airways in healthy subjects and chronic lung disease: a bronchoscopic study. Eur Respir J 1997; 10: 1137-1144

${ }^{6}$ Monsó E, Ruiz J, Rosell A et al. Bacterial infection in chronic obstructive pulmonary disease. A study of stable and exacerbated outpatients using the protected specimen brush. Am J Respir Crit Care Med 1995; 152: $1316-1320$

${ }^{7}$ Riise GC, Larsson S, Larrson P et al. The intrabronchial microbial flora in chronic bronchitis patients: a target for $\mathrm{N}$-acetylcysteine therapy? Eur Respir J 1994; 7: 94-101

${ }^{8}$ Soler N, Ewig S, Torres A et al. Airway inflammation and bronchial microbial patterns in stable patients with chronic obstructive pulmonary disease (COPD). Eur Respir J 1999; 14: 1015-1022

${ }^{9}$ Zalacain R, Sobradillo V, Amilibia J et al. Predisposing factors to bacterial colonization in chronic obstructive pulmonary disease. Eur Respir J 1999; 13: $343-348$

10 Patel IS, Seemungal TAR, Wilks M et al. Relationship between bacterial colonization and the frequency, character, and severity of COPD exacerbations. Thorax 2002; 57: 759-764

${ }^{11}$ Seemungal T, Harper-Owen R, Bhowmik A et al. Respiratory viruses, symptoms, and inflammatory markers in acute exacerbations and stable chronic obstructive pulmonary disease. Am J Respir Crit Care Med 2001; 164: 1618 - 1623

12 Sethi S, Evans N, Grant BJB et al. New strains of bacteria and exacerbations of chronic obstructive pulmonary disease. N Engl J Med 2002; 347: $465-471$

${ }^{13}$ Donaldson GC, Seemungal TAR, Bhowmik A et al. Relationship between exacerbation frequency and lung function decline in chronic obstructive pulmonary disease. Thorax 2002; 57: 847-852

${ }^{14}$ Wilkinson TMA, Patel IS, Wilks M et al. Airway bronchial load and FEV decline in patients with chronic obstructive pulmonary disease. Am J Respir Crit Care Med 2003; 167: 1090 - 1095

${ }^{15}$ Anzueto A, Niederman MS, Tillotson GS. Etiology, susceptibility, and treatment of acute bacterial exacerbations of complicated chronic bronchitis in the primary care setting: ciprofloxacin $750 \mathrm{mg}$ b.i.d. versus clarithromycin $500 \mathrm{mg}$ b.i.d. Bronchitis study group. Clin Ther 1998; 20: 885-900

${ }^{16}$ Fagon JY, Chastre J, Trouillet JL et al. Characterisation of distal microflora during acute exacerbations of chronic bronchitis. Am Rev Respir Dis 1990; 142: 1004- 1008

17 Soler N, Torres A, Ewig S et al. Bronchial microbial patterns in severe exacerbations of chronic obstructive pulmonary disease (COPD) requiring mechanical ventilation. Am J Respir Crit Care Med 1998; 157: $1498-1505$

${ }^{18}$ Beaty CD, Grayston JT, Wang SP et al. Chlamydia pneumoniae, strain TWAR, infection in patients with chronic obstructive pulmonary disease. Am Rev Respir Dis 1991; 144: 1408-1410 
${ }^{19}$ Blasi F, Legnani D, Lombardo WM et al. Chlamydia pneumoniae infection in acute exacerbations of chronic obstructive pulmonary disease (COPD). Eur Respir J 1993; 6: 19-22

${ }^{20}$ Mogulkoc N, Karakurt S, Isalska B et al. Acute purulent exacerbation of chronic obstructive pulmonary disease and Chlamydia pneumoniae infection. Am J Respir Crit Care Med 1999; 160: 349-353

${ }^{21}$ McHardy VU, Inglis JM, Calder MA. A study of infective and other factors in exacerbations of chronic bronchitis. Br J Dis Chest 1980; 74: $228-238$

22 McNamara MJ, Philipas IA, Williams OB. Viral and mycoplasma pneumoniae infections in exacerbations of chronic lung disease. Am Rev Respir Dis 1969; 100: 19-24

${ }^{23}$ Smith CB, Golden CA, Kanner RE et al. Association of viral and mycoplasmal infections with respiratory illness in patients with chronic obstructive pulmonary diseases. Am Rev Respir Dis 1980; 121: $225-232$

${ }^{24}$ Buscho RO, Saxtan D, Shultz PS et al. Infection with viruses and Mycoplasma pneumoniae during exacerbations of chronic bronchitis. J Infect Dis 1978; 137: $377-383$

${ }^{25}$ Lieberman D, Lieberman D, Shmarkov O et al. Serological evidence of Legionella species infection in acute exacerbation of COPD. Eur Respir J 2002; 19: $392-397$

${ }^{26}$ Rohde G, Wiethege A, Borg I et al. Respiratory viruses in exacerbations of chronic obstructive pulmonary disease requiring hospitalisation: a case-control study. Thorax 2003; 58: 37-42

27 Smith CB, Golden CA, Klauber MR et al. Interactions between viruses and bacteria in patients with chronic bronchitis. J Infect Dis 1976; 134: $552-561$

${ }^{28}$ Saint S, Bent S, Vittinghoff E et al. Antibiotics in chronic obstructive pulmonary disease exacerbations: a meta-analysis. JAMA 1995; 273: 957-960

${ }^{29}$ Sachs APE, Koeter GH, Groenier KH et al. Changes in symptoms, peak expiratory flow, and sputum flora during treatment with antibiotics of exacerbations in patients with chronic obstructive pulmonary disease in general practice. Thorax 1995; 50: $758-763$

${ }^{30}$ Nouria S, Marghli S, Belghith M et al. Once daily oral ofloxacin in chronic obstructive pulmonary disease exacerbation requiring mechnical ventilation: a randomized placebo-controlled trial. Lancet 2001; 358: 2020-2025

${ }^{31}$ Anthonisen N, Manfreda J, Warren CPW et al. Antibiotic therapy in excerbations of chronic obstructive pulmonary disease. Ann Intern Med 1987; 106: 196-204

32 Jorgensen AF, Coolidge J, Pedersen PA et al. Amoxicillin in treatment of acute uncomplicated exacerbations of chronic bronchitis. Scand J Prim Health 1992; 16: 7-11

${ }^{33}$ Nicotra MB, Rivera M, Awe RJ. Antibiotic therapy of acute exacerbations of chronic bronchitis. Ann Intern Med 1982; 97: 18-21

${ }^{34}$ Stockley RA, O'Brien C, Pye A et al. Relationship of sputum colour to nature and outpatient management of acute exacerbations of COPD. Chest 2000; 117: $1638-1645$

${ }^{35}$ Ball P, Harris JM, Lowson D et al. Acute infective exacerbations of chronic bronchitis. Quart J Med 1995; 88: 61 -68

${ }^{36}$ Dewan NA, Rafique S, Kanwar B et al. Acute exacerbation of COPD. Factors associated with poor treatment outcome. Chest 2000; 117: $662-671$

${ }^{37}$ Adams S, Melo J, Luther M et al. Antibiotics are associated with lower relapse rates in outpatients with acute exacerbations of COPD. Chest 2000; 117: 1345 - 1352

38 Wilson R, Allegra L, Huchon G et al. Short-term and long-term outcomes of moxifloxacin compared to standard antibiotic treatment in acute exacerbations of chronic bronchitis. Chest 2004; 125: 953 - 964

${ }^{39}$ Seneff MG, Wagner DP, Wagner RP et al. Hospital and 1-year survival of patients admitted to intensive care units with acute exacerbation of chronic obstructive pulmonary disease. JAMA 1995; 274: 1852 - 1857

${ }^{40}$ Gunen H, Hacievliyagil SS, Kosar F et al. Factors affecting survival of hospitalised patients with COPD. Eur Respir J 2005; 26: 234 - 241

${ }^{41}$ European Study on Community-acquired pneumonia (ESOCAP) Commitee. Guidelines for management of adult community-acquired lower respiratory tract infections. Eur Respir J 1998; 11: 986 - 991

${ }^{42}$ Eller J, Ede A, Schaberg T et al. Infective exacerbations of chronic obstructive pulmonary disease. Relation between bacteriologic etiology and lung function. Chest 1998; 113: $1542-1548$

${ }^{43}$ Miravitlles M, Espinosa C, Fernandez-Laso E et al. Relationship between bacterial flora in sputum and functional impairment in patients with acute exacerbations of COPD. Chest 1999; 116: 40-46
${ }^{44}$ Brauers J, Bagel S, Kresken M. Ambulant erworbene Atemwegsinfektionen. Resistenzen bakterieller Erreger gegenüber Antibiotika in Deutschland. Med Welt 2004; 10: 327-331

${ }^{45}$ Miravittles M, Torres A. No more equivalence trials for antibiotics in exacerbations of COPD, please. Chest 2004; 125: 811-813

${ }^{46}$ Chodosh S, Schreurs A, Siami G et al. Efficacy of oral ciprofloxacin vs. clarithromycin for treatment of acute bacterial exacerbations of chronic bronchitis. The Bronchitis Study Group. Clin Infect Dis 1998; 27: $730-738$

47 Wilson R, Schentag JJ, Ball P et al. A comparison of gemifloxacin and clarithromycin in acute exacerbations of chronic bronchitis and longterm clinical outcomes. Clin Ther 2002; 24: 639-652

${ }^{48}$ Lode H, Eller J, Linnhoff A et al. Levofloxacin versus clarithromycin in COPD exacerbation: focus on exacerbation-free interval. Eur Respir J 2004; 24: $947-953$

${ }^{49}$ Ruhe JJ, Hasbun R. Streptococcus pneumoniae bacteremia: duration of previous antibiotic use and association with penicillin resistance. Clin Infect Dis 2003; 36: $1132-1138$

${ }^{50}$ Paul M, Benuri-Silbiger I, Soares-Weiser K et al. Beta lactam monotherapy versus beta lactam-aminoglycoside combination therapy for sepsis in immunocompetent patients: systematic review and meta-analysis of randomised trials. BMJ 2004; 328: 668-673

51 Safdar N, Handelsman J, Maki DG. Does combination antimicrobia therapy reduce mortality in Gram-negative bacteraemia? A metaanalysis. Lancet Infect Dis 2004; 4: 519-527

\section{Information}

Das Deutsche Zentralkomitee zur Bekämpfung der Tuberkulose (DZK) hat seinen 29. Informationsbericht veröffentlicht. Er enthält auf 100 Seiten aktuelle Beiträge zur weltweiten Tuberkulosesituation und ihren möglichen Auswirkungen auf Deutschland sowie einen Artikel zur Tuberkulosesituation in Deutschland 2003, der in Zusammenarbeit mit dem Robert Koch-Institut erstellt wurde. Weiterhin enthält der Jahresbericht eine Übersicht mit dem Titel „Beitrag des Öffentlichen Gesundheitsdienstes zur Kontrolle der Tuberkulose in Deutschland“ vom Arbeitskreis Tuberkulose im Fachausschuss Infektionsschutz des Bundesverbandes der Ärzte des Öffentlichen Gesundheitsdienstes sowie einen Beitrag aus dem Nationalen Referenzzentrum für Mykobakterien in Borstel, „Aktuelle mikrobiologische TuberkuloseDiagnostik“, und eine Zusammenfassung zur Begutachtung der Tuberkulose als Berufskrankheit von Dr. A. Nienhaus bei der BGW. Daneben finden sich Übersichten über die Aktivitäten und Publikationen des DZK und vieles mehr.

Der DZK-Bericht stellt in seiner neuen Form für den TuberkuloseInteressierten eine informative Lektüre zu wichtigen aktuellen Fragen dar. Er kann zum Preis von $€ 15,00$ zzgl. Porto beim DZK bestellt werden.

Deutsches Zentralkomitee zur

Bekämpfung der Tuberkulose

Lungenklinik Heckeshorn

Zum Heckeshorn 33

14109 Berlin

Tel.: 030/ 81022435

Fax: 030/81022286 